\title{
Nuclear Medicine Training: What Now?
}

\author{
David Mankoff and Daniel A. Pryma \\ Division of Nuclear Medicine, Department of Radiology, Perelman School of Medicine, University of Pennsylvania, Philadelphia, \\ Pennsylvania
}

Although the multidisciplinary nature of nuclear medicine (NM) and clinical molecular imaging is a key strength of the specialty, the breadth of disciplines involved in the practice of NM creates challenges for education and training. The evolution of NM science and technology - and the practice of clinical molecular imaging and theranostics - has created a need for changes in the approach to specialty training. The broader U.S. community of imaging physicians has been slow to accept this change, in good part due to historical divides between the NM and nuclear radiology (NR) communities. In this Journal of Nuclear Medicine Hot Topics discussion, we review the historical pathways to training; discuss the training needs for the modern practice of NM, clinical molecular imaging, and radionuclide therapy; and suggest a path forward for an approach to training that matches the needs of the evolving clinical specialty.

\section{HISTORICAL APPROACHES TO TRAINING AND CERTIFICATION}

The growth of radionuclide imaging and therapy practice in the late 1960s and 1970s led to the need to define training and certification for the young specialty of nuclear medicine (NM). Early NM practitioners came from specialties focused on physiology and laboratory assays such as endocrinology and pathology/laboratory medicine. As advances in radioisotope technology provided more anatomically resolute images, NM also garnered interest from the radiology community. A series of discussions and compromises led to 2 distinct training and certification pathways (1):

1. Nuclear Medicine (NM): A distinct medical specialty encompassing broad training in diagnostic radionuclide procedures, radionuclide imaging, and radionuclide therapy. This specialty has a dedicated residency pathway (Nuclear Medicine Residency) and an independent certification board, the American Board of Nuclear Medicine (ABNM), founded in 1971 and falling under the aegis of the American Board of Medical Specialties.

2. Nuclear Radiology (NR): A subspecialty of diagnostic radiology focused on the diagnostic application of radionuclide imaging. This training pathway has a specific fellowship (Nuclear Radiology) open to physicians who are board-eligible/-certified in Diagnostic Radiology by the American Board of Radiology (ABR) leading to a subspecialty certificate in NR. NR certification is administered by the ABR.

Received Aug. 1, 2017; revision accepted Aug. 9, 2017.

For correspondence or reprints contact: David A. Mankoff, University of Pennsylvania, 116 Donner, HUP, 3400 Spruce St., Philadelphia, PA 19104. E-mail: david.mankoff@uphs.upenn.edu

Published online Aug. 17, 2017.

COPYRIGHT (C) 2017 by the Society of Nuclear Medicine and Molecular Imaging. DOI: 10.2967/jnumed.117.190132
Although these specialties and certification pathways were in theory distinct, they have evolved to become highly overlapping. In the past, both NM and NR focused on the diagnostic aspects of radionuclide imaging, whereas only NM provided training and certification in radionuclide therapy and the application of radiotracer imaging, including imaging combined with stress testing, to cardiovascular disease (i.e., nuclear cardiology). At the inception of the specialties, $\mathrm{NM}$, but not NR, included in-depth training in radionuclide therapy and thus only certification in NM held deemed status as a radioisotope authorized user (AU) by the national Nuclear Regulatory Commission. Physicians without diagnostic radiology training could be certified in NM (but not NR) after completing an NM residency. Physicians trained in diagnostic radiology (which includes a minimum of 4 mo of training in NM during radiology residency) can qualify for certification in radioisotope specialties by undertaking a 1-y fellowship in an accredited NR fellowship to sit the examination for an ABR NR certificate, or they could undertake a 1-y NM training in an accredited NM residency to qualify for ABNM certification in NM (2).

As if this weren't confusing enough already, changes in the NR curriculum in $2012(3,4)$ added radionuclide therapy, leading to more overlap with NM training and certification. The revised NR curriculum and certification now leads to deemed status as an AU for diagnostic procedures and selected radionuclide therapy procedures (oral $\mathrm{Na}^{131}$ I therapy), but is still not quite as broad as NM training, which encompasses a greater range of therapy procedures as well as nuclear cardiac stress testing. The change in the NR fellowship requirements led the ABNM to decide in 2012 to accept NR trainees to the ABNM certification examination, provided that they had completed all aspects of the NM curriculum and ABNM certification prerequisites - mostly related to more extensive training and experience in radionuclide therapy and cardiac stress testing. At the University of Pennsylvania, since 2012, all of our successful NR fellows have pursued ABNM certification, supported by an NR fellowship that includes the required components of NM training.

An additional and important complexity arises from the fact that, in most U.S. centers, specific training and certification in NM-beyond a limited 4-mo exposure during diagnostic radiology residency - is not required for practitioners who are certified in diagnostic radiology to practice NM. Changes in AU-deemed status by the Nuclear Regulatory Commission enabled ABR Diagnostic Radiology diplomates to supervise diagnostic procedures and to perform oral radioiodine therapy for both benign and malignant diseases, and qualified radiation oncology diplomates to serve as AUs for the broad range of isotope therapy previously reserved for ABNM diplomates.

The state of training, certification, and practice for NM and its subcomponents remains in this hybrid and highly confusing mix to this day. Physicians practicing radionuclide therapy and imaging 
in the United States have a wide range of expertise and training in radioisotope procedures ranging from 4 mo to $3 \mathrm{y}$ or more.

\section{EVOLUTION OF THE SPECIALTY—AND ITS TRAINING NEEDS}

Despite a mix of views on appropriate training and certification required for NM practice by the various groups involved, and the chaos of multiple training and certification pathways, the specialty of NM evolved and thrived. Pure radioisotope imaging has given way to increasingly hybrid imaging-PET/CT, SPECT/CT, and PET/MR $(5,6)$. Furthermore, imaging protocols have become increasingly standardized with less patient-specific interaction and procedural customization. Finally, even in the absence of hybrid devices, the advent of electronic medical records and PACS systems has fostered appropriate and nearly ubiquitous use of correlative anatomic imaging in the interpretation of radionuclide imaging procedures. In this evolution-which has been fostered by both the NM and the NR communities (5) - the practice of radionuclide imaging in the absence of anatomic imaging has become increasingly rare, creating a need for NM practitioners with in-depth training in anatomic imaging methods- that is, diagnostic radiology. In addition, clinical molecular imaging is moving beyond radionuclide imaging to include optical methods, targeted ultrasound contrast, and possibly molecularly targeted MR or CT contrast methods $(7,8)$, requiring training in molecular imaging approaches beyond radioisotope imaging.

Radionuclide therapy has also evolved and is moving away from a practice predominantly focused on radioiodine treatment of thyroid diseases to a much broader range of theranostic agents designed to treat a variety of diseases. Radionuclide therapy increasingly targets more lethal cancers for which treatment may be pushed to toxicity limits, akin to the practice of radiation oncology and medical oncology (9). The historical pure NM physician can no longer practice diagnostic nuclear imaging without more in-depth training in anatomic diagnostic imaging. Conversely, the pure diagnostic imager can no longer encompass the breadth of clinical molecular imaging and theranostics in the emerging practice of NM without more in-depth training in radioisotope imaging and therapy, clinical patient management, and molecular imaging science. The evolution of our specialty has increased the need for combined, multispecialty training. Clearly, training for NM needs to evolve with the practice.

\section{NM TRAINING: WHERE DO WE GO FROM HERE?}

Fans of the late Dr. Seuss might remember a story called "The Zax" (10), where a north-going and a south-going Zax, both marching straight head, came face to face in the sparsely populated Prairie of Prax. Both refused to budge, stopping the forward progress for both of them. Time led to population growth in the Prairie of Prax, causing other Prairie dwellers to build roads around and over the 2 stubborn Zax, who remained stuck in their tracks as the world around them progressed. We, the current NM and NR communities (along with a bit of help from radiation oncologists and cardiologists), are the imaging world's Zax, and the broader medical community will soon build roads aroundand over-us, driven by the desire to move advances in molecular imaging and theranostics into modern medical practice. How can we avoid the fate that befell the Zax?

Perhaps there is light shining at the edge of the molecular imaging and theranostic prairie. Molecular imaging and theranostics, both largely done with radioisotope techniques for the foreseeable future, are increasingly relevant in the era of precision medicine. There is an important niche for these techniques at the center of critical patient care decisions and, thus, there will be a need for translational and clinical scientists to continue to move these techniques forward and for clinicians to offer them in widespread practice. Recognizing a need for combined training in radiology and NM for the molecular imager of the future, and taking advantage of a now subspecialty-friendly diagnostic radiology residency - the NM and NR communities banded together to create a 16-mo NM training (in either NM or NR) pathway embedded into a 4-y diagnostic radiology residency (11). This pathway generates well-trained molecular imagers who are proficient in clinical molecular imaging and theranostics and have sufficient expertise in anatomic imaging to take full advantage of hybrid imaging methods and correlative imaging. Furthermore, 16-mo pathway residents can go on to traditional radiology fellowships for further subspecialty training to gain additional specialty training that meets the needs of advanced practices and academic centers providing advanced subspecialty molecular imaging, for example, PET/CT. Alternatively, the traditional 1-y NR or NM fellowship can be combined with tailored fourth year training (e.g., an oncologic imaging pathway) or a dedicated research year to yield physicians with unique specializations that match the needs of rapidly advancing academic and specialty NM practices and translational molecular imaging research. At the University of Pennsylvania, working closely with our Diagnostic Radiology Residency leadership, we have taken advantage of these pathways to yield physicians with training in NM plus neuroradiology, breast imaging, musculoskeletal imaging, pediatrics, oncologic imaging, and basic/translational molecular imaging research. The result has been astounding, attracting some of the best and brightest members of our residency program to pursue NM-and, indeed, attracting some of the best and brightest medical students to our diagnostic radiology residency program. The result has been a cadre of outstanding early career NM physicians prepared to practice the molecular imaging of the future and to carry on the research needed to keep the specialty at the cutting edge of medical practice.

Advances in the field of molecular imaging and theranostics also indicate the need for cross-fertilization in highly specialized areas of the practice such as nuclear cardiology and radionuclide therapy/ theranostics. Some leading examples of cardiologists who understand the biology and pathophysiology of cardiovascular disease and who are cross-trained as molecular imagers who understand the science and breadth of methodology in molecular imaging have clearly enriched the practice of cardiac molecular imaging. Finding a way to train cardiologists focused on aspects of cardiac molecular imaging will continue to elevate the practice through a diversity of inputs. The current model for levels of certification in the highly focused subspecialty of cardiovascular molecular imaging provides a nice paradigm for this goal $(12,13)$. Similarly, radiation oncologistswho, by the nature of their training are comfortable with patient management in the face of therapeutic toxicities, understand the biology and toxicity of therapeutic radiation, and have considerable imaging training for treatment planning purposes-can gain much from expanded knowledge and experience of theranostic agent kinetics and dosimetry to create comprehensive and highly tailored approaches to radionuclide therapy. One can envision a training pathway for combined radiation oncology and NM akin to the 16-mo embedded diagnostic radiology practice to create a radiation oncology specialty practitioner with advanced training in image-guided therapy and theranostics. 


\section{SUMMARY AND FUTURE DIRECTIONS}

Radionuclide molecular imaging and therapy has a bright future and is poised to play a pivotal role in critical patient management decisions in the era of precision medicine (14). Will we, the NM practitioners of the present, get stuck in our tracks facing our radiology (and cardiology and radiation oncology) brethren and refusing to budge while the rest of medicine builds road around, over, and through us? Or will the story end differently (as in some other Dr. Seuss stories) where we all work together to train the NM practitioners of the future who are broadly prepared to take molecular imaging and theranostics into the future? A common fear among current NM physicians is that we will lose our specialty if we move to joint training with radiology and other specialties. The reality is much to the contrary: we will surely lose our specialty if we continue to stand our ground without budging on the approach to combined training and certification. Progress in molecular imaging and theranostics will surely bypass our intransigence if we stay stuck in our current path. Alternatively, we can embrace collaborative, multidisciplinary training to ensure our specialty its well-deserved place as a key component of the future practice of medicine. Our Dutch colleagues have taken this approach by creating a nuclear-focused pathway in the context of radiology training (15). It is time for the United States to follow this lead and expand it to other specialties such as cardiology and radiation oncology.

\section{DISCLOSURE}

No potential conflict of interest relevant to this article was reported.

\section{ACKNOWLEDGMENTS}

We thank the Journal leadership for the chance to contribute this Hot Topics editorial. We note that the views expressed are those of the authors and are not intended to reflect the views of the JNM, SNMMI, or ABNM.

\section{REFERENCES}

1. Gordon LL, Royal HD, Mankoff DA. ABNM celebrates 40th anniversary. J Nucl Med. $2011 ; 52: 17 \mathrm{~N}-18 \mathrm{~N}$

2. Delbeke D, Graham M, Royal H, et al. Conjoint statement of the SNM, ACNM, and $\mathrm{ABNM}$ on credentialing and delineation of privileges for therapeutic procedures using radiopharmaceuticals. J Nucl Med. 2011;52:323-326.

3. Royal HD. Diagnostic radiology and nuclear radiology program requirements changes challenged. J Nucl Med. 2012;53:16N.

4. Ziessman H. Nuclear medicine residency vs nuclear radiology fellowship. J Nucl Med. 2012;53:13N.

5. Delbeke D, Royal HD, Frey KA, Graham MM, Segall GM. SNMMI/ABNM joint position statement on optimizing training in nuclear medicine in the era of hybrid imaging. J Nucl Med. 2012;53:1490-1494.

6. Frey KA, Royal HD, Di Carli MF, et al. ABNM position statement: nuclear medicine professional competency and scope of practice. J Nucl Med. 2011;52: 994-997.

7. Mankoff DA. A definition of molecular imaging. J Nucl Med. 2007;48:18N, $21 \mathrm{~N}$.

8. Weissleder R, Schwaiger MC, Gambhir SS, Hricak H. Imaging approaches to optimize molecular therapies. Sci Transl Med. 2016;8:355ps16.

9. Loke KS, Padhy AK, Ng DC, Goh AS, Divgi C. Dosimetric considerations in radioimmunotherapy and systemic radionuclide therapies: a review. World $\mathrm{J} \mathrm{Nucl}$ Med. 2011;10:122-138.

10. Seuss D. The Sneetches and Other Stories. New York: Random House; 1961.

11. Oates ME, Guiberteau MJ. Adoption of the 16-month American Board of Radiology pathway to dual board certifications in nuclear radiology and/or nuclear medicine for diagnostic radiology residents. Acad Radiol. 2014;21:1348-1356.

12. Dilsizian V, Arrighi JA, Cohen RS, Miller TD, Solomon AJ, Udelson JE. COCATS 4 Task Force 6: training in nuclear cardiology. J Am Coll Cardiol. 2015;65:1800-1809.

13. Narula J, Chandrashekhar YS, Dilsizian V, et al. COCATS 4 Task Force 4: training in multimodality imaging. J Am Coll Cardiol. 2015;65:1778-1785.

14. Mankoff DA, Farwell MD, Clark AS, Pryma DA. Making molecular imaging a clinical tool for precision oncology: a review. JAMA Oncol. 2017;3:695-701.

15. van Schaik JPJ, Bennink RJ. Integrated residency in radiology and nuclear medicine in The Netherlands. J Nucl Med. 2017;58:9N-11N. 